DOI: 10.12731/2658-6649-2020-12-2-73-83

УДК 57.083.32

\title{
АКТУАЛЬНОСТЬ АЛЛЕРГИЧЕСКИХ ПОРАЖЕНИЙ ПИЩЕВАРИТЕЛЬНОГО ТРАКТА У ДЕТЕЙ
}

\author{
Давыдова А.Н., Шапошникова Н.Ф., \\ Маркелов В.В., Юрченко О.А.
}

Широкая распространённость и постоянный рост аллергических заболеваний, ассочиированных с пищеварительной системой у детей, остаются одной из наиболее актуальных проблем современной гастроэнтерологии. В развитых странах более 60\% пачиентов с пищевой аллергией имеют гастроинтестинальные расстройства. В большинстве исследований доказана первоочередная роль нарушений пищеварительного барьера в генезе аллергодерматозов, бронхиальной астмы, хронической крапивницы и т.n. Развитие аллергических реакций в желудочно-кишечном тракте является результатом иммунного ответа на различные антигенные структуры.

Ключевые слова: пищевая аллергия; аллергодерматоз; хроническая крапивница; эозинофильный эзофагит.

\section{THE RELEVANCE OF ALLERGIC LESIONS OF THE DIGESTIVE TRACT IN CHILDREN}

\author{
Davydova A.N., Shaposhnikova N.F., \\ Markelov V.V., Yurchenko O.A.
}

The widespread and constant growth of allergic diseases associated with the digestive system in children remains one of the most pressing problems of modern gastroenterology. In developed countries, more than $60 \%$ of patients with food allergies have gastrointestinal disorders. Most studies have proven the primary role of digestive barrier disorders in the genesis of allergic dermatoses, bronchial asthma, chronic urticaria, etc. The development of allergic reactions in the gastrointestinal tract is the result of an immune response to various antigenic structures.

Keywords: food allergy; allergic dermatosis; chronic urticarial; eosinophilic esophagitis. 


\section{Введение}

К факторам риска, которые провоцируют развитие аллергических состояний, относятся генетическая предрасположенность (на возникновение гастроинтестинальных аллергических заболеваний влияет, в первую очередь, наследственная предрасположенность к поражениям желудочно-кишечного тракта), гестозы, возникающие во время беременности, ухудшение экологической обстановки, курение родителей, незрелость системы пищеварения у новорожденных, несвоевременное введение прикормов (и очень раннее, и позднее), незрелые механизмы регуляции местного иммунитета, многочисленные эффекты материнских гормонов, высокая проницаемость слизистой оболочки ЖКТ.

Именно в этих слизистых начинается локальная тканевая реакция после попадания пищевого аллергена, что провоцирует включение слизистых оболочек пищеварительного тракта в иммунный процесс.

Иммунная система желудочно-кишечного тракта выполняет отбор основного спектра питательных компонентов, необходимых для развития ребенка и предотвращает запуск патологического восприятия белковых субстанций, который и проявляется аллергическими расстройствами. В большинстве случаев иммунная реакция к белкам не развивается из-за способности системы иммунитета формировать толерантность к пище. Практически 100 \% пищевых белков при неповрежденном слизистом барьере ЖКТ и физиологическом полостном пищеварении подвергаются деградации и усвоению, и лишь незначительное их количество (менее $2 \%$ ) всасывается в неизмененном виде.

Гастроэнтерологические симптомы пищевой аллергии многообразны и зависят от вида и дозы аллергена, возраста пациента, степени и глубины поражения ЖКТ. Морфологическая основа клинических симптомов в большинстве случаев - иммунное воспаление с преимущественно эозинофильной инфильтрацией тканей (если нет иных причин - паразитарных инвазий, новообразований, системных заболеваний, патологии сосудистого звена и воспалительных заболеваний кишечника). Эозинофильные поражения ЖКТ многочисленны: эозинофильный эзофагит, эозинофильный гастроэнтерит, эозинофильный энтерит, эозинофильный колит, эозинофильный проктит и прочие патологические состояния.

\section{Обсуждение полученных результатов}

У здоровых детей эозинофильные клетки присутствуют во многих органах и системах - пищеварительном тракте, селезёнке, лимфатических узлах, тимусе, и способствуют защите организма от паразитов и глист- 
ных инвазий. В ЖКТ они встречаются в собственной пластинке слизистой оболочки практически всех отделов (желудок, тонкая и толстая кишки), пищевод является исключением. Нормальное содержание эозинофилов различно и зависит от отдела пищеварительной системы. Если количество эозинофильных клеток выше физиологической нормы, следует заподозрить эозинофильное поражение.

Разнообразные клинические формы повреждений пищеварительного тракта при гастроинтестинальной аллергии имеют особенности - по механизму возникновения, возрасту пациента (в котором манифестирует данная проблема), характеру течения, диагностической и лечебной тактике. Клинические проявления зависят от локализации поражения и глубины эозинофильной инфильтрации. Среди эозинофильных поражений ЖКТ у детей условно выделяют слизистую, мышечную и серозную формы (в связи с тем, что в рутинной практике специалистов часто встречаются смешанные формы, с различной степенью выраженности отдельных симптомов).

Наиболее распространенной является слизистая форма, которая проявляется болями в животе, тошнотой, рвотой и диареей. Симптомы при этой форме зависят от уровня поражения пищеварительной системы. Дисфагия и боли за грудиной - наиболее характерные жалобы при эозинофильном эзофагите; боли в эпигастрии и рвота - обычные проявления эозинофильного поражения желудка. Повреждение тонкой кишки характеризуется расстройством стула, сопровождается клиникой синдрома мальабсорбции, а при масштабном поражении происходит активная потеря белка с развитием гипоальбуминемии и появлением отеков. Поражение толстой кишки характеризуется наличием дизентериеподобного синдрома с большим количеством слизи и крови в каловых массах.

Повреждение при мышечной форме эозинофильной гастроэнтеропатии может иметь локальный или генерализованный характер. Наиболее активно в патологический процесс вовлекается антральная часть желудка, что сопровождается выраженным утолщением желудочной стенки, и проявляется клинической картиной обструктивного синдрома. У детей раннего возраста эта форма напоминает клиническую картину гипертрофического пилоростеноза. При поражении кишечника отмечается сегментарное или диффузное утолщение кишечной стенки, что требует проведения дифференциальной диагностики с болезнью Крона. Эозинофильное поражение аппендикса клинически напоминает течение острого аппендицита или обострение хронического.

В последние годы во всем мире отмечается значительный рост числа пациентов с эозинофильным эзофагитом как среди детского, так и среди 
взрослого населения. Эозинофильный эзофагит диагностируется в любом возрасте. Международные исследования показывают, что частота встречаемости этой проблемы сопоставима с другими иммуннопатологическими состояниями, например, такими как воспалительные заболевания кишечника.

Эозинофильный эзофагит (ЭоЭ) - это хроническое иммунное антигенопосредованное воспалительное заболевание пищевода, характеризующееся выраженной эозинофильной инфильтрацией слизистой оболочки пищевода, клинически выражающееся дисфункцией пищевода (дисфагией). В методических рекомендациях Европейской академии аллергологии и клинической иммунологии по лечению пищевой аллергии и анафилаксии (European Academy of Allergy and Clinical Immunology, EAACI; Food Allergy and Anaphylaxi sGuidelines) приводится практически аналогичное определение ЭоЭ. Акцент делается на клинических симптомах болезни, связанных с нарушением функционального состояния пищевода.

Большинство авторов сходится во мнении, что у детей от рождения до шестилетнего возраста с эозинофильным поражением пищевода наиболее часто развиваются неспецифические симптомы, которые вызывают определенные сложности при кормлении (рвота, регургитация или отказ от пищи), в результате чего ребенок начинает отставать в физическом развитии.

В клинической картине у детей старше шестилетнего возраста и взрослых пациентов преобладают жалобы на ощущение «комка в горле», поперхивания и другие признаки нарушенного глотания, изжогу, боли в груди. А у детей раннего возраста, как отмечалось выше, преобладают другие симптомы: срыгивания, тошнота, рвота, анорексия, боли в животе, раздражительность и беспокойство. Клиническая и эндоскопическая картины эозинофильного эзофагита неспецифичны и, в основном, не отличаются от «классического» эзофагита. При диагностике эозинофильного поражения пищевода мало помогают исследование уровня общего IgE, который часто остается в пределах нормы или слегка повышен; увеличение количества эозинофилов в периферической крови необязательно, а результаты скарификационных проб часто отрицательны. На мысль об аллергопатологии наводит резистентность к традиционной медикаментозной терапии и отсутствие эффекта от хирургической коррекции. Такие пациенты часто имеют осложненный аллергологический анамнез и отягощенную наследственность по атопическим состояниям.

Верифицировать диагноз можно только при эндоскопическом исследовании с обязательным морфологическим подтверждением. В отличие от «классического» рефлюкс-эзофагита эозинофильный эзофагит редко 
связан с ГЭР. Характерный признак - поражение пищевода на всём его протяжении, а не только дистального отдела.

При гистологическом исследовании биопсийного материала слизистой пищевода выявляются эозинофильная инфильтрация с участками Т-клеточной активации в слизистом и подслизистом слоях, гипертрофия папилярной и базальной зоны. Морфологические изменения ограничиваются только пищеводом и не выявляются других отделах пищеварительного тракта. Эозинофильная инфильтрация иногда может отмечаться и при гастро-эзофагеальной рефлюксной болезни, но её уровень незначительный и не превышает 5 эозинофилов в поле зрения, в то время как при эозинофильном эзофагите она достигает 15-25 клеток в поле зрения. Правильность диагноза эозинофильного поражения пищевода подтверждается выраженным эффектом элиминационной (с удалением из рациона всех видов аллергенов) или элементной диет, а также эффективностью системных или топических стероидных препаратов.

Алгоритм обследования детей и подростков при подозрении на эозинофильный эзофагит подробно описанных в методических рекомендациях ESPGHAN 2014.

Цель лечения эозинофильного эзофагита - устранение симптомов и восстановление физиологической эндоскопической и гистологической картины.

Для исключения действия пищи как причинно-значимого фактора в развитии эозинофильной реакции разработаны различные варианты элиминационных диетических рационов. Замечено, что эффективность таких диет у детей значительно выше, чем у взрослых, поэтому на современном этапе ограничение и/или исключение потребления отдельных продуктов (значимых аллергенов) в обязательном порядке рекомендуется всем детям с данной патологией.

В настоящее время у больных с эозинофильным эзофагитом изучена эффективность трех диетических режимов:

- элементная диета (аминокислотная формула);

- таргетная (целевая, индивидуально ориентированная) элиминационная диета с исключением продуктов, вызывающих аллергическую реакцию у конкретного пациента;

- эмпирическая элиминационная диета с исключением из рациона продуктов, являющихся доказанными или вероятными триггерами аллергических реакций.

Согласно алгоритму, у ребенка с подтвержденным диагнозом эозинофильного эзофагита необходимо оценить аллергоанамнез и результаты тестов к 
пищевым аллергенам (если таковые проводились). С учетом этих данных проводится выбор вариантов терапевтической коррекции - диета и/или глюкокортикостероиды. Известно, что пациенты без отягощенного аллергологического анамнеза хуже отвечают на диетотерапию и требуют проведения медикаментозной терапии. У части пациентов могут возникать сезонные обострения, вызванные воздушными, пылевыми, пыльцевыми аллергенами. В этих случаях необходимо выявить провоцирующие факторы (аэроаллергены), и рекомендовать в полном объеме диетическую коррекцию и топические глюкокортикостероиды для предотвращения обострения заболевания.

Топические и системные глюкокортикостероиды показали высокую эффективность в достижении ремиссии эозинофильного эзофагита независимо от возраста пациентов. Потенциальные побочные эффекты и токсичность при длительном применении системных препаратов, в частности, флутиказонапропионата и будесонида, привели к назначению топических глюкокортикостероидов с нарушением рекомендаций утвержденной инструкции к лекарственным средствам (off-label).

Высокая эффективность данной группы лекарственных препаратов в терапии эозильнофильного эзофагита обусловлена их ярко выраженной способностью подавлять синтез факторов роста эозинофилов и вызывать апоптоз эозинофилов.

Топические глюкокортикостероиды являются препаратами первой линии после так называемого «тестового» лечения ингибиторами протонной помпы. Эти препараты хорошо переносятся пациентами (в том числе, детьми); их основными побочными эффектами являются кандидоз и герпетическая инфекция полости рта и пищевода (но возникают они, как правило, при длительном использовании). Однако следует учитывать, что назначение других групп лекарственных препаратов (ввиду отсутствия их доказанной эффективности) в настоящее время невозможно.

\section{Заключение}

Эозинофильный эзофагит является хронической проблемой, соответственно, сохраняется и у взрослых людей. Тем не менее, трансформации в другие заболевания пищеварительного тракта (гиперэозинофильный синдром, пищевод Барретта, аденокарциному пищевода) не происходит, что доказано результатами длительных клинических наблюдений. Воспалительный процесс не распространяется за пределы пищевода, что обеспечивает благоприятный прогноз для жизни пациентов с эозинофильным эзофагитом. 


\section{Список литературы}

1. Chitkara D.K., Camilleri M., Zinsmeister A.R. et al. Gastricsensory and motor dysfunction in adolescents with functional dyspepsia. J Pediatr. 2005; 146:500-505.

2. Drossman D.A., Hasler W.L. Rome IV - Functional GI Disorders: Disorders of Gut-Brain Interaction. Gastroenterology. 2016;150(6):1257-1261.

3. Hyams J.S., Di Lorenzo C., Saps M. et al. Childhood Functional Gastrointestinal Disorders: Child/Adolescent. Gastroenterology. 2016;150:1456-1468.

4. Matricardi PM, Kleine-Tebbe J, Hoffmann HJ, Valenta R, Hilger C, Hofmaier S, Aalberse RC, Agache I, Asero R, Ballmer-Weber B, Barber D, Beyer K, Biedermann T, Bilò MB, Blank S, Bohle B, Bosshard PP, Breiteneder H, Brough HA, Caraballo L, Caubet JC, Crameri R, Davies JM, Douladiris N, Ebisawa M, EIgenmann PA, Fernandez-Rivas M, Ferreira F, Gadermaier G, Glatz M, Hamilton RG, Hawranek T, Hellings P, Hoffmann-Sommergruber K, Jakob T, Jappe U, Jutel M, Kamath SD, Knol EF, Korosec P, Kuehn A, Lack G, Lopata AL, Mäkelä M, Morisset M, Niederberger V, Nowak-Węgrzyn AH, Papadopoulos NG, Pastorello EA, Pauli G, Platts-Mills T, Posa D, Poulsen LK, Raulf M, Sastre J, Scala E, Schmid JM, Schmid-Grendelmeier P, van Hage M, van Ree R, Vieths S, Weber R, Wickman M, Muraro A, Ollert M. EAACI Molecular Allergology User's Guide. Pediatr Allergy Immunol. 2016 May; 27 Suppl 23:1-250.

5. Muraro A, Werfel T, Hoffmann-Sommergruber K, Roberts G, Beyer K, Bindslev-Jensen C, Cardona V, Dubois A, duToit G, Eigenmann P, Fernandez Rivas M, Halken S, Hickstein L, Høst A, Knol E, Lack G, Marchisotto MJ, Niggemann B, Nwaru BI, Papadopoulos NG, Poulsen LK, Santos AF, Skypala I, Schoepfer A, Van Ree R, Venter C, Worm M, VliegBoerstra B, Panesar S, de Silva D, Soares-Weiser K, Sheikh A, Ballmer-Weber BK, Nilsson C, de Jong NW, Akdis CA; EAACI Food Allergy and Anaphylaxis Guidelines Group. EAACI food allergy and anaphylaxis guidelines: diagnosis and management of food allergy. Allergy. 2014 Aug;69(8):1008-25.

6. Papadopoulou A, Koletzko S, Heuschkel R, et al. Management guidelines of eosinophilic esophagitis in childhood. J Pediatr Gastroenterol Nutr. 2014;58(1):107-118. doi: 10.1097/MPG.0b013e3182a80be1.

7. Rabinowitz Simon S., Goli Sridhar, Xu Jiliu, Zhang Xuchen, Nicastri Anthony, Anderson Virginia, StefanovDimitre G., Schwarz Steven M. Lack of correlation between obesity and gastroesophageal reflux disease (GERD) in a pediatric cohort // Open Journal of Pediatrics. 2013. Vol. 3, №4. P. 317-323.

8. Schwarz S., Hebra A. Gastroesophageal Reflux // eMedicine, Last Updated: 06 May 2013. http://emedicine.medscape.com/article/930029-overview 
9. Баранов А.А., Намазова-Баранова Л.С., Новик Г.А., и др. Протокол ведения детей с пищевой аллергией. Клинические рекомендации Союза педиатров России. М.: Педиатръ, 2016. 52 с.

10. Беляева И.А., Макарова С.Г. Функциональные нарушения желудочно-кишечного тракта у детей: новые аспекты диагностики и лечения // Вопросы современной педиатрии. 2016. Т.15(2). С. 212-214.

11. Ивашкин В.Т., Баранская Е.К., Трухманов А.С., Кайбышева В.О. Эозинофильный эзофагит. Учебное пособие для врачей. М.: АИСПИ РАН; 2013. $80 \mathrm{c}$.

12. Кешишян Е.С., Бердникова Е.К., Хавкин А.И. Функциональные нарушения желудочно-кишечного тракта у детей раннего возраста // Практика педиатра. 2012. №9. С. 12-16.

13. Кильдиярова Р.Р., Гурова М.М. Функциональные гастроинтестинальные нарушения у детей: аспекты диагностики и лечения // Фарматека. 2018. T.2(355). С. 36-42.

14. Клиническая диетология детского возраста. Руководство для врачей. 2-е издание. Под редакцией Т.Э. Боровик, К.С. Ладодо. Москва, МИА, 2015, $718 \mathrm{c}$.

15. Макарова С.Г., Намазова-Баранова Л.С., Вишнева Е.А., Геворкян А.К., Алексеева А.А., Петровская М.И. Актуальные вопросы диагностики пищевой аллергии в педиатрической практике. Вестник РАМН. 2015; 1: 41-46.

16. Намазова-Баранова Л.С. Аллергия у детей: от теории к практике. М.: Союз педиатров России. 2010-2011. 668 с.

17. Приворотский В.Ф., Луппова Н.Е. Кислотозависимые заболевания у детей (клиническая картина, диагностика, лечение). Учеб. пособие. 2-е изд., испр. и доп. СПб.: Изд. дом СПбМАПО, 2005. 136 с.

\section{References}

1. Chitkara D.K., Camilleri M., Zinsmeister A.R. et al. Gastricsensory and motor dysfunction in adolescents with functional dyspepsia. J Pediatr. 2005;146:500505.

2. Drossman D.A., Hasler W.L. Rome IV - Functional GI Disorders: Disorders of Gut-Brain Interaction. Gastroenterology. 2016;150(6):1257-1261.

3. Hyams J.S., Di Lorenzo C., Saps M. et al. Childhood Functional Gastrointestinal Disorders: Child/Adolescent. Gastroenterology. 2016;150:1456-1468.

4. Matricardi PM, Kleine-Tebbe J, Hoffmann HJ, Valenta R, Hilger C, Hofmaier S, Aalberse RC, Agache I, Asero R, Ballmer-Weber B, Barber D, Beyer K, Biedermann T, Bilò MB, Blank S, Bohle B, Bosshard PP, Breiteneder H, Brough 
HA, Caraballo L, Caubet JC, Crameri R, Davies JM, Douladiris N, Ebisawa M, EIgenmann PA, Fernandez-Rivas M, Ferreira F, Gadermaier G, Glatz M, Hamilton RG, Hawranek T, Hellings P, Hoffmann-Sommergruber K, Jakob T, Jappe U, Jutel M, Kamath SD, Knol EF, Korosec P, Kuehn A, Lack G, Lopata AL, Mäkelä M, Morisset M, Niederberger V, Nowak-Węgrzyn AH, Papadopoulos NG, Pastorello EA, Pauli G, Platts-Mills T, Posa D, Poulsen LK, Raulf M, Sastre J, Scala E, Schmid JM, Schmid-Grendelmeier P, van Hage M, van Ree R, Vieths S, Weber R, Wickman M, Muraro A, Ollert M. EAACI Molecular Allergology User's Guide. Pediatr Allergy Immunol. 2016 May; 27 Suppl 23:1-250.

5. Muraro A, Werfel T, Hoffmann-Sommergruber K, Roberts G, Beyer K, Bindslev-Jensen C, Cardona V, Dubois A, duToit G, Eigenmann P, Fernandez Rivas M, Halken S, Hickstein L, Høst A, Knol E, Lack G, Marchisotto MJ, Niggemann B, Nwaru BI, Papadopoulos NG, Poulsen LK, Santos AF, Skypala I, Schoepfer A, Van Ree R, Venter C, Worm M, VliegBoerstra B, Panesar S, de Silva D, Soares-Weiser K, Sheikh A, Ballmer-Weber BK, Nilsson C, de Jong NW, Akdis CA; EAACI Food Allergy and Anaphylaxis Guidelines Group. EAACI food allergy and anaphylaxis guidelines: diagnosis and management of food allergy. Allergy. 2014 Aug;69(8):1008-25.

6. Papadopoulou A, Koletzko S, Heuschkel R, et al. Management guidelines of eosinophilic esophagitis in childhood. J Pediatr Gastroenterol Nutr. 2014;58(1):107118. doi: 10.1097/MPG.0b013e3182a80be1.

7. Rabinowitz Simon S., Goli Sridhar, Xu Jiliu, Zhang Xuchen, Nicastri Anthony, Anderson Virginia, StefanovDimitre G., Schwarz Steven M. Lack of correlation between obesity and gastroesophageal reflux disease (GERD) in a pediatric cohort. Open Journal of Pediatrics. 2013. Vol. 3, №4, pp. 317-323.

8. Schwarz S., Hebra A. Gastroesophageal Reflux. eMedicine, Last Updated: 06 May 2013. http://emedicine.medscape.com/article/930029-overview

9. Baranov A.A., Namazova-Baranova L.S., Novik G.A. et al. Protokol vedeniya detey s pishchevoy allergiey. Klinicheskie rekomendatsii Soyuza pediatrov Rossii [Protocol for the management of children with food allergies. Clinical recommendations of the Union of Pediatricians of Russia]. M.: Pediatr, 2016. 52 p.

10. Belyaeva I.A., Makarova S.G. Voprosy sovremennoy pediatrii. 2016. V.15(2), pp. 212-214.

11. Ivashkin V.T., Baranskaya E.K., Trukhmanov A.S., Kaybysheva V.O. Eozinofil'nyy ezofagit. Uchebnoe posobie dlya vrachey [Eosinophilic esophagitis. A manual for doctors]. M.: AISPI RAN; 2013. 80 p.

12. Keshishyan E.S., Berdnikova E.K., Khavkin A.I. Praktika pediatra. 2012. №9, pp. 12-16. 
13. Kil'diyarova R.R., Gurova M.M. Farmateka. 2018. V.2(355), pp. 36-42.

14. Klinicheskaya dietologiya detskogo vozrasta. Rukovodstvo dlya vrachey [Clinical dietetics of childhood. A guide for doctors]. Ed. T.E. Borovik, K.S. Ladodo. Moskva, MIA, 2015, 718 p.

15. Makarova S.G., Namazova-Baranova L.S., Vishneva E.A., Gevorkyan A.K., Alekseeva A.A., Petrovskaya M.I. Vestnik RAMN. 2015; 1: 41-46.

16. Namazova-Baranova L.S. Allergiya u detey: ot teorii k praktike. M.: Soyuz pediatrov Rossii. 2010-2011. 668 s.

17. Privorotskiy V.F., Luppova N.E. Kislotozavisimye zabolevaniya u detey (klinicheskaya kartina, diagnostika, lechenie) [Acid-dependent diseases in children (clinical presentation, diagnosis, treatment)]. SPb.: Izd. dom SPbMAPO, 2005. 136 p.

\section{ДАННЫЕ ОБ АВТОРАХ}

Давыдова Альбина Николаевна, доцент, кандидат медицинских наук Волгоградский Государственный Медицинский Университет пл. Павших Боризов, 1, г. Волгоград, 400131, Российская Федерация dr.a.davydova@gmail.com

Шапошникова Наталья Федоровна, доцент, кандидат медицинских наук Волгоградский Государственный Медицинский Университет пл. Павших Боризов, 1, г. Волгоград, 400131, Российская Федерация nataliashaposhnikova1957@gmail.com

\section{Маркелов Вадим Владиславович, студент}

Волгоградский Государственный Медицинский Университет пл. Павиих Боричов, 1, г. Волгоград, 400131, Российская Федераичи vadim.911@mail.ru

\section{Юрченко Олеся Алексеевна, студент}

Волгоградский Государственный Медицинский Университет пл. Павших Боријов, 1, г. Волгоград, 400131, Российская Федерация olesya.yurchenko.97@mail.ru

\section{DATA ABOUT THE AUTHORS}

Davydova Albina Nikolaevna, Associate Professor, Candidate of Medical Sciences Volgograd State Medical University

1, Pavshikh Bortsov, Volgograd, 400131, Russian Federation dr.a.davydova@gmail.com 
Shaposhnikova N Natalya Fedorovna, Associate Professor, Candidate of Medical Sciences

Volgograd State Medical University

1, Pavshikh Bortsov, Volgograd, 400131, Russian Federation nataliashaposhnikova1957@gmail.com

Markelov Vadim Vladislavovich, student Volgograd State Medical University 1, Pavshikh Bortsov, Volgograd, 400131, Russian Federation vadim.911@mail.ru

Yurchenko Olesya Alekseevna, student

Volgograd State Medical University

1, Pavshikh Bortsov, Volgograd, 400131, Russian Federation olesya.yurchenko.97@mail.ru 